\title{
Composição e distribuição da fauna de Ephemeroptera (Insecta) em área de transição Cerrado-Amazônia, Brasil
}

\author{
Yulie Shimano $^{1}$, Helena S. R. Cabette ${ }^{2}$, Frederico F. Salles ${ }^{3} \&$ Leandro Juen $^{4}$
}

\begin{abstract}
1. Programa de Pós-graduação em Ecologia e Conservação, Universidade do Estado de Mato Grosso, Caixa Postal 08, $78690-000$ Nova Xavantina, MT, Brasil. (yulie.bio@gmail.com)

2. Universidade do Estado de Mato Grosso, Caixa Postal 08, 78690-000 Nova Xavantina, MT, Brasil.

3. Centro Universitário Norte do Espírito Santo, Universidade Federal do Espírito Santo, São Mateus, ES, Brasil.

4. Programa de Pós-graduação em Ecologia e Evolução, Universidade Federal de Goiás, Caixa Postal 24241, 74.690-970 Goiânia, GO, Brasil.
\end{abstract}

\begin{abstract}
Composition and distribution of Ephemeroptera (Insecta) in Cerrado-Amazônia transition area, Brazil. The objective of this study was to respond if exist a pattern on the spatial distributions of Ephemeroptera nymphs in different streams and rivers from Suiá-Miçú Basin, MT, and how the streams are classifying according to the species composition. Were sample 12 streams and rivers, in three periods of the year. Were collected 1,356 individuals, distributed in seven families, 31 genus and 42 species and/or morphospecies. The most abundant locals were semi-lentics and with few shaded suggering that most light in the system offer, allochthonous material and autochthonous material like food resources. The greater estimates richness was found in lotic places, emphasizing that the Ephemeroptera presents greater richness in these places, once water current is essential for organic matter transporting. So much in the grouping analysis (TWINSPAM) as in the ordination (DCA) was observed the separation of lotic and semi-lentic environments as for species composition. It had a gradient in the composition similarity of lotic and small streams for lotic and big stream and for last, semilentics. The results of this study show that the physic structure of aquatic environments affect species composition. In that way, retreat of riparian vegetation and streams dam up can take significant losses in the diversity.
\end{abstract}

KEYWORDS. Aquatic insects, Suiá-Miçú River Basin, lotic and semi-lentic environments, nymphs.

RESUMO. O objetivo deste trabalho foi responder se existe padrão na distribuição espacial das ninfas de Ephemeroptera em diferentes córregos e rios da bacia do Rio Suiá-Miçú, MT, e como os córregos estudados estão classificados de acordo com a composição de espécies. $\mathrm{Na}$ coleta foram amostrados 12 córregos e rios, em três períodos do ano. Foram coletados 1356 indivíduos, distribuídos em sete famílias, 31 gêneros e 42 espécies e/ou morfoespécies. Os locais mais abundantes foram ambientes semi-lênticos, com pouco sombreamento no leito, sugerindo que a maior entrada de luz disporia, além do material alóctone, material autóctone como fonte de alimento. A maior riqueza de espécie e/ou morfoespécie, estimada, foi em ambientes lóticos enfatizando que os Ephemeroptera apresentam maior riqueza nestes locais uma vez que a correnteza é essencial para transportar a matéria orgânica particulada. Tanto na análise de agrupamento (TWINSPAM) como na ordenação (DCA) observou-se a separação entre ambientes lóticos e semi-lênticos quanto a composição de espécies. Percebeu-se um gradiente na similaridade de composição dos ambientes lóticos de pequeno porte para ambientes lóticos de grande porte e, por fim, os semi-lênticos. Os resultados deste estudo demonstram que a estrutura física dos ambientes aquáticos afeta a composição de espécies. Dessa forma, a retirada das matas ciliares e o represamento dos córregos podem levar à perdas significativas na diversidade.

PALAVRAS-CHAVE. Insetos aquáticos, bacia do rio Suiá-Miçú, ambiente lótico e semi-lêntico, ninfas.

Conhecer a diversidade de espécies e o seu padrão de distribuição espacial tem sido um dos focos centrais em ecologia nos últimos tempos (Downes \& ReICH, 2008). De acordo com Melo (2008), identificar a diversidade de espécies de uma área é fundamental para a compreensão da natureza, conservação de recursos naturais ou até mesmo para a recuperação dos ecossistemas degradados, permitindo restabelecer os serviços ecossistêmicos anulados pelos impactos ambientais.

No Brasil, várias iniciativas têm sido realizadas no intuito de identificar e caracterizar a estrutura das comunidades. No entanto, os estudos ainda são bastantes incipientes devido às grandes dimensões do país e sua elevada biodiversidade (MitTERMEIER et al., 2005). Quando consideramos as comunidades de invertebrados dos ecossistemas límnicos, o maior número de trabalhos é das regiões sudeste, sul e norte, refletindo a distribuição dos pesquisadores (e.g. KIKUCHI \& UIEDA, 1998; FRANCISCHETTI et al., 2004; CRISCI-BISPO et al., 2007; Fidelis et al., 2008; Siegloch et al., 2008). Na região centro-oeste, pouco se tem publicado (e.g. BISPO et al., 2006; Bispo \& Oliveira, 2007; CABETte et al., 2010).

Essa lacuna no conhecimento sobre os macroinvertebrados é preocupante, devido sua importância nos ecossistemas aquáticos, uma vez que desempenham papel fundamental na transformação da matéria orgânica, no fluxo de energia e na ciclagem de nutrientes (DodDs, 2002). Dentre os macroinvertebrados bentônicos, a ordem Ephemeroptera se destaca como um dos principais grupos, principalmente por possuírem representantes em todos os grupos funcionais, serem abundantes e habitarem a maior parte dos hábitats disponíveis em ambientes lênticos e lóticos (BARBERJAMES et al., 2008).

Os Ephemeroptera compõem um pequeno grupo de insetos aquáticos, com 10 famílias, 65 gêneros e 223 espécies registradas no Brasil. No estado de Mato Grosso são registrados apenas cinco famílias (Baetidae, Leptophlebiidae, Leptohyphidae, Caenidae e Polymitarcyidae), 21 gêneros e 27 espécies (SALLES, 2010). 
As ninfas de Ephemeroptera são utilizadas como bioindicadores de qualidade de água, uma vez que possuem alta sensibilidade às alterações na estrutura física e qualidade da água dos córregos (ROSENBERG \& RESH, 1993).

Por outro lado, Mato Grosso é um dos estados campeões em desmatamento e no número de focos de incêndios, liderando, juntamente com o Pará, os principais responsáveis pelo avanço do desmatamento do bioma Amazônico (SchwARTZMAn \& ZiMMERMAN, 2005).

Esse mesmo cenário de devastação é registrado na bacia do rio Suiá-Miçú, afluente do rio Xingu, que se encontra em uma área de transição entre a Floresta Amazônica e o Cerrado. Apesar da existência de uma grande área preservada dentro do Parque Indígena do Xingu (Neu, 2006), isso não assegura a proteção dessa bacia, uma vez que as principais nascentes e cabeceiras estão situadas fora da área protegida e sofrem forte pressão do avanço agropecuário. De acordo com ScHWARTZMAN \& ZimMERMAN (2005), o avanço das plantações de soja, criação de gado, extração de madeira e a pesca predatória estão entre as principais ameaças desse ecossistema.

Sem a vegetação ripária, as chuvas têm causado o assoreamento dos cursos d'água, além de carrear os poluentes (insumos agrícolas e defensivos químicos como pesticidas e herbicidas) para dentro de córregos e rios, contaminando as bacias inteiras e alterando toda a cadeia alimentar (Moulton \& Souza, 2006). Tais alterações podem resultar em extinções locais, bem como, mudanças drásticas nas abundâncias das espécies. Situação essa que pode ser agravada ainda mais quando se trata de um grupo com grande sensibilidade a mudanças ambientais como os Ephemeroptera.

$\mathrm{O}$ objetivo deste trabalho consistiu em verificar o padrão de distribuição espacial das ninfas de Ephemeroptera em diferentes córregos e rios da bacia do rio Suiá-Miçú, MT e como os córregos estudados estão classificados, de acordo com a composição de espécies.

\section{MATERIAL E MÉTODOS}

O estudo foi conduzido ao longo da bacia do rio Suiá-Miçú, nos municípios de Querência, Ribeirão Cascalheira e Canarana, na porção leste do estado de Mato Grosso. Foram selecionados 12 pontos de coleta ao longo da bacia, abrangendo todas as fisionomias entre os paralelos $11^{\circ} 49^{\prime} 50^{\prime}$ 'S e $13^{\circ} 15^{\prime} 45^{\prime}$ 'S e os meridianos $51^{\circ} 53$ '20"W e 52 21'27'W (Fig. 1). Os ambientes variaram de $1^{\text {a }}$ à $6^{\mathrm{a}}$ ordem (de acordo com a classificação de STRAHLER, 1957), porém, devido às diferenças entre os corpos d'água, eles foram classificados de acordo com as características físicas dos ambientes. Ambientes como o córrego Lúcio ( $2^{\mathrm{a}}$ ordem - CRL), rio Piabanha ( $2^{\mathrm{a}}$ ordem - RIPB), rio Suiá-Miçú, ponto um (2a ordem - RISU1) e ponto dois ( $3^{\mathrm{a}}$ ordem - RISU2) são lóticos de pequeno porte, variando de um a oito metros de largura. $\mathrm{O}$ rio Suiá-Miçú no ponto três ( $6^{\mathrm{a}}$ ordem - RISU3) e o rio Suiazinho (4 ${ }^{\mathrm{a}}$ ordem - RISUZ) são maiores, tendo mais do que $10 \mathrm{~m}$ de largura, também lóticos. Os córregos Sucuri ( $2^{\mathrm{a}}$ ordem - CRSRI), Brejão ( $1^{\mathrm{a}}$ ordem - CRBJ) e o rio Darro ( $5^{\text {a }}$ ordem - RID) são ambientes extensos, alagados e semilênticos, conhecidos como varjões ou brejos, com predominância de buritis (Mauritia flexuosa-Aracaceae). Por fim, três locais foram considerados alterados: o rio Betis, ponto dois ( $1^{\mathrm{a}}$ ordem - RIBET2) e o córrego Brejo ( $1^{\mathrm{a}}$ ordem - CRTB) são locais represados (semi-lênticos) com pisoteio frequente de gado, enquanto que o rio Betis no ponto um ( $1^{\mathrm{a}}$ ordem - RIBET1) é lótico, de pequeno porte, mas está sobre influência antrópica, a apenas $20 \mathrm{~m}$ abaixo da represa.

De acordo com RATTER et al. (1978), a região apresenta clima tropical sazonal com estação seca de maio a outubro e chuvosa de novembro a abril, classificada como subtipo Savana (Aw) e com microrregiões do subtipo Monções (Am) e Tropical Chuvoso (A) segundo classificação de Köppen. A precipitação média é 1370 mm e temperatura entre $17^{\circ} \mathrm{C}$ e $32,7^{\circ} \mathrm{C}$. As coletas foram realizadas em três estações, na seca (setembro/2007), início de chuva (dezembro/2007) e vazante (maio/2007).

A amostragem dos imaturos de Ephemeroptera foi realizada através da metodologia de transecto (metodologia adaptada de FerreirA-Peruquetti \& DE

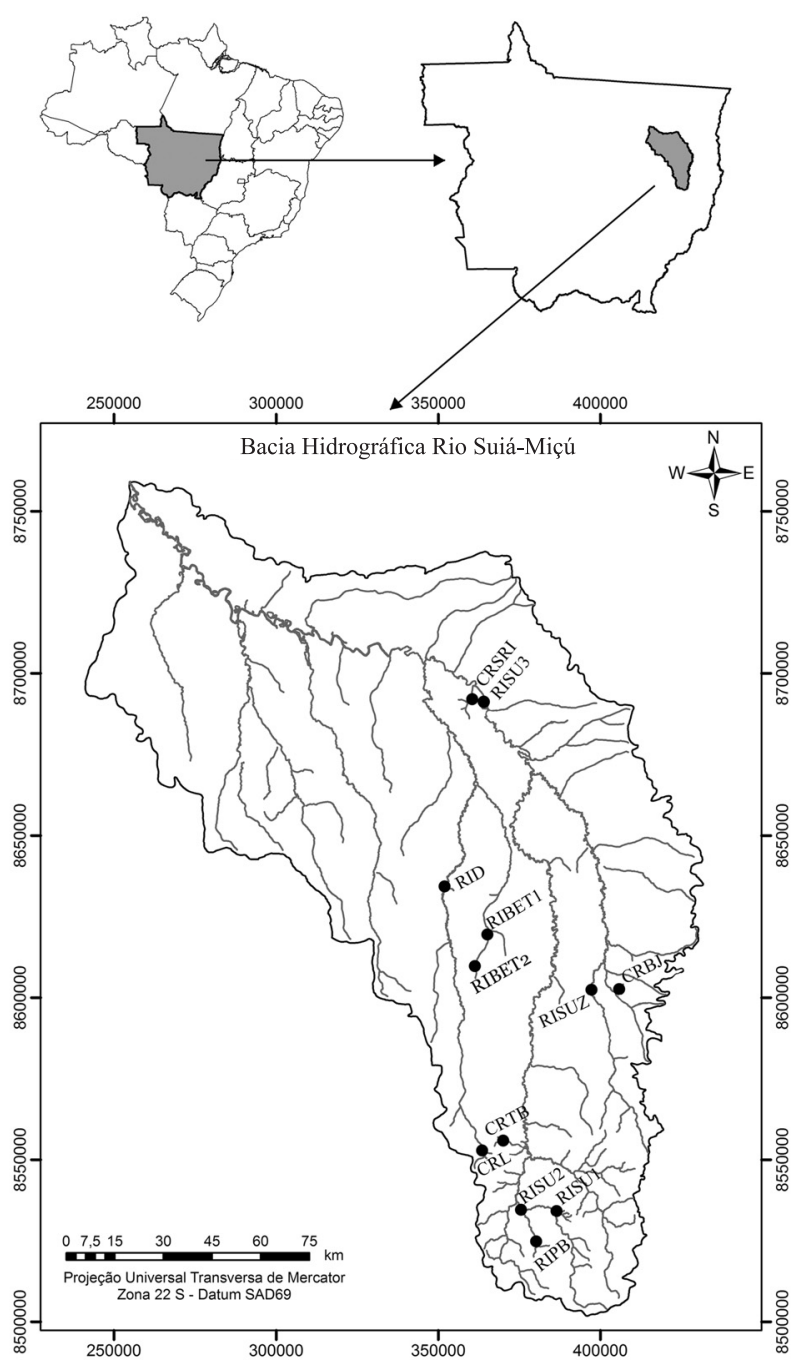

Figura 1. Pontos de coleta de Ephemeroptera imaturos na bacia do rio Suiá-Miçú, MT (CRSRI, córrego Sucuri; RISU3, rio Suiá-Miçú local 3; RIBET2, rio Betis represado; RIPB, rio Piabanha; RIBET1, rio Betis na mata; RISU2, rio Suiá-Miçú local 2; CRBJ, córrego Brejão; CRTB, nascente do Brejo na transição; RISU1, rio SuiáMiçú local 1; RID, rio Darro; RISUZ, rio Suiazinho; CRL, córrego Lúcio). 
MARCO, 2002). Para tal, foram demarcados $100 \mathrm{~m}$ na margem direita dos corpos d'água, subdivididos em 20 segmentos de cinco metros cada um. As amostras foram obtidas através de um coador de $18 \mathrm{~cm}$ de diâmetro e malha de $0,05 \mathrm{~mm}$ (rapiché), replicando-se três vezes uma porção aleatórias dos substratos presentes em cada segmento do centro para margem dos córregos. O material coletado foi triado em campo com o auxílio de pinças entomológicas e bandejas brancas, os espécimes foram separados e fixados em álcool a $85 \%$.

Para a identificação dos espécimes foram utilizadas chaves taxonômicas de Domínguez et al. (2006) e Dias et al. (2007). Os espécimes foram depositados na Coleção Zoobotânica "James Alexander Ratter", na Universidade do Estado de Mato Grosso, Campus de Nova Xavantina.

Considerando que a riqueza de espécies observada é frequentemente um estimador viciado para a riqueza de espécies real (SANTos, 2003), a riqueza de espécies foi estimada. Como todas as amostras constituíram o mesmo tamanho e esforço amostral, a riqueza de espécie e/ou morfoespécie foi estimada através do estimador Jackknife de $1^{a}$ ordem (Jack 1), que dá maior peso às espécies raras (aquelas que ocorreram em apenas uma amostra) (Burnham \& Overton, 1978). Mesmo que o método subestime a riqueza da comunidade, isto não constitui um grande problema, uma vez que o que em geral se espera das estimativas de riqueza é obter parâmetros para comparações entre inventários (SAntos, 2003). Foi realizada a rarefação padronizada por amostra, pois conforme argumenta CouRTEMANCH (1996) não se pode comparar amostras padronizadas por uma certa quantidade de indivíduos, visto que se perde muita informação quanto à abundância/densidade das espécies nas comunidades. A técnica de rarefação utilizada foi a "baseada em momentos" (Mao Tau) (Gotelli \& Colwell, 2001), que elimina completamente a necessidade de réplica, permite a comparação direta de riqueza entre amostras e é menos intensiva computacionalmente do que o Jackknife (Colwell et al., 2004). Ambas as técnicas de estimativas foram calculadas no programa EstimateS 7.5.0 (Colwell, 2005).

Com a finalidade de identificar possíveis agrupamentos dos riachos através do compartilhamento de espécies, foi aplicada a análise de classificação do TWINSPAN (Two-Way Indicator Species Analysis) (HILL, 1979), nesta análise são apontadas espécies indicadoras do agrupamento, que são as espécies que foram exclusivas de cada lado da divisão. Para ordenar os 12 pontos de amostragem, visando agrupar aqueles mais similares em relação à composição e presença/ausência das espécies, foi utilizada a análise de correspondência destendenciada com remoção do efeito do arco (Detreated Correspondence Analysis - DCA) (HILL \& GAUCH, 1980).

$\mathrm{O}$ uso de duas análises multivariadas aparentemente similares se justifica devido a distinção de objetivos de cada análise. Os métodos de ordenação têm como objetivo principal revelar mudanças contínuas e suaves na estrutura das comunidades, enquanto que métodos de classificação têm como objetivo revelar grupos de amostras e a interligação entre os grupos (MELo \& HEPP, 2008).

\section{RESULTADOS}

Foram coletados 1356 indivíduos, distribuídos em sete famílias (Baetidae, Caenidae, Coryphoridae, Euthyplociidae, Leptohyphidae, Leptophlebiidae e Polymirtacyidae), 31 gêneros e 42 espécies e/ou morfoespécies. As famílias mais abundantes foram Leptophlebiidae $(n=522)$ e Baetidae $(n=300)$, enquanto as famílias Coryphoridae $(n=14)$ e Euthyplociidae $(n=16)$ foram as que tiveram a menor abundância (Tab. I).

As espécies mais abundantes foram Caenis cuniana Froehlich, $1969(\mathrm{n}=155)$, Fittkaulus sp. $(\mathrm{n}=107)$, Simothraulopsis sp. $(\mathrm{n}=105)$ e Callibaetis sp.1 $(\mathrm{n}=103)$, que também foram bem distribuídas, estando presente em pelo menos metade dos locais amostrados. Ulmeritoides flavopedes (Spieth, 1943) foi a espécie que apresentou a maior amplitude de distribuição, ocorrendo em dez dos 12 pontos de coleta, não sendo observada apenas nos locais CRL e CRTB. Apesar da alta abundância do gênero Campsurus Eaton, 1868 (n=119), os indivíduos foram considerados como uma única espécie nas análises, em virtude de dificuldades taxonômicas, como descrições incompletas ou figuras inadequadas, e uma grande similaridade morfológica entre as espécies.

Com relação à distribuição das espécies nos ambientes estudados, o RID, CRSRI e CRBJ, ambientes alagados e semi-lênticos, apresentaram as maiores abundâncias amostradas $(n=259,226$ e 227, respectivamente). Nesses ambientes, Callibaetis sp. 1 e sp. 2 foram as espécies mais abundantes. Os ambientes que apresentaram as menores abundâncias foram os ambientes com interferências antrópicas, RIBET1 $(n=11)$, CRTB $(n=36)$ e RIBET2 ( $n=57)$.

A riqueza das espécies/morfoespécies apresentou o mesmo padrão em ambas as técnicas de estimativas, apesar dos valores gerados pelo Jackknife serem maiores (Fig. 2). Os locais mais ricos foram ambientes lóticos de médio e grande porte (RISU1, RISUZ e RISU2) enquanto que os menos ricos também foram os ambientes que apresentam influência antrópica (CRTB, RIBET1 e RIBET2). Os ambientes de pequeno porte e semi-lênticos apresentaram valores de riquezas intermediárias.

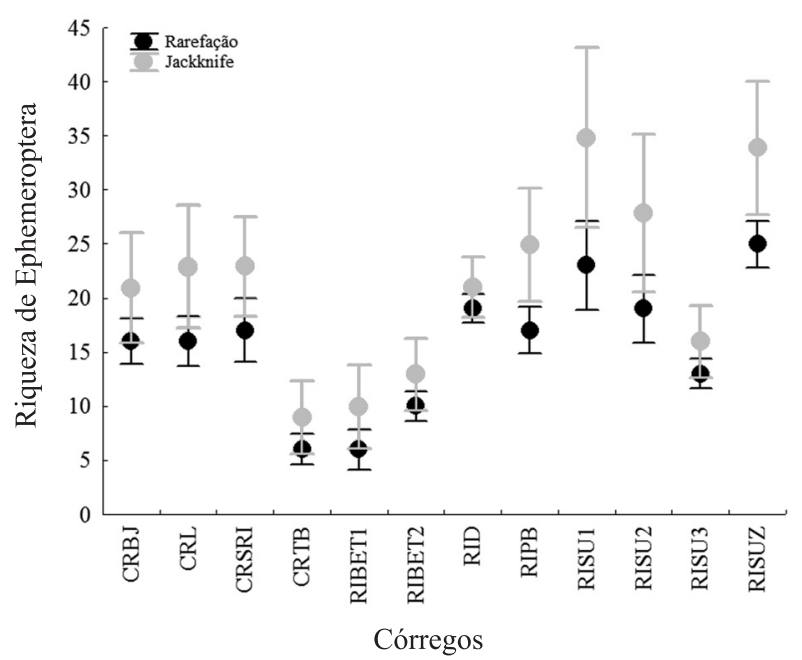

Figura 2. Riquezas estimadas de morfoespécies de Ephemeroptera nos córregos e rios da bacia do rio Suiá-Miçú, MT, coletados em 2007 e 2008 (as barras representam intervalo de confiança de 95\%). 
Na classificação dos córregos e rios, o TWINSPAN indicou na primeira divisão a separação dos ambientes classificados como lóticos (RISU1, RISU2, RISU3, RISUZ, CRL e CRPB) dos ambientes semi-lênticos alterados (CRTB e RIBET2) e não alterados (RID, CBBJ, CRSRI) (Fig. 3). Caenis cuniana foi exclusiva dos ambientes semilênticos (espécie indicadora), enquanto que os ambientes lóticos não tiveram espécies indicadoras.
Na segunda divisão, no primeiro grupo (ambientes lóticos), o RISU3 se separou dos demais e Amanahyphes saguassu Salles \& Molineri, 2006 foi a espécie indicadora desse subgrupo. No segundo grupo (ambientes semilênticos), o RIBET1 se separou dos outros ambientes, sendo que Cryptonympha copiosa Lugo-Ortiz \& McCafferty, 1998 foi a indicadora dos demais ambientes semi-lênticos.

Tabela I. Espécies e/ou morfoespécies de Ephemeroptera coletados em córregos da bacia do rio Suiá-Miçú-MT em 2007 e 2008 , abundância e riqueza observada por córrego (siglas correspondentes aos locais, ver figura 1).

\begin{tabular}{|c|c|c|c|c|c|c|c|c|c|c|c|c|c|}
\hline \multirow[t]{2}{*}{ Espécies/Morfoespécies } & \multicolumn{13}{|c|}{ Localidade } \\
\hline & RISU1 & RISU2 & RISU3 & RIBET1 & RIBET2 & RIPB & RISUZ & RID & CRSRI & CRBJ & CRTB & CRL & Total \\
\hline Amanahyphes saguassu Salles \& Molineri, 2006 & 2 & 7 & & & & 2 & 5 & & & 1 & & 12 & 29 \\
\hline Americabaetis alphus Lugo-Ortiz \& McCafferty, 1995 & 1 & 1 & & & 8 & & & & 2 & & & & 12 \\
\hline Apobaetis fuizai Salles \& Lugo-Ortiz, 2002 & & & 1 & & & & & & & & & & 1 \\
\hline Askola sp. & 4 & & & & & 2 & 1 & & & & & 2 & 9 \\
\hline Asthenopus cf. curtus & & & & & 2 & & & 30 & 18 & 17 & & & 67 \\
\hline Aturbina georgei Lugo-Ortiz \& McCafferty, 1996 & & 1 & 3 & & & & & 12 & 4 & & & & 20 \\
\hline Aturbina sp. n. & 4 & 3 & 4 & & & 1 & 6 & & & & & 1 & 19 \\
\hline Brasilocaenis irmeli Puthz, 1975 & & & 4 & 1 & & & & & & & & & 5 \\
\hline Brasilocaenis sp. & & & 17 & 1 & & 2 & 4 & & 1 & & & & 25 \\
\hline Caenis cuniana Froehlich, 1969 & & & & 4 & 20 & & & 3 & 45 & 73 & 10 & & 155 \\
\hline Caenis fitkaui Malzacher, 1986 & & & & & 4 & & & 1 & 23 & 3 & & & 31 \\
\hline Caenis pflugfelderi Malzacher, 1990 & 1 & 1 & 4 & 3 & & 2 & 1 & 3 & & & & & 15 \\
\hline Callibaetis sp. 1 & & & & 1 & 12 & & 1 & 13 & 26 & 35 & 14 & 1 & 103 \\
\hline Callibaetis sp. 2 & & & & & & & & 30 & 1 & 3 & & & 34 \\
\hline Campsurus spp. & 23 & 17 & 26 & & 6 & 6 & 13 & 27 & 1 & & & & 119 \\
\hline Campylocia sp. & 5 & & & & & 2 & 2 & & & & & 7 & 16 \\
\hline Cloeodes auwe Salles \& Batista, 2004 & 4 & & & & & & 7 & & & & & & 11 \\
\hline Cloeodes sp. & 1 & & & & & & & & & & & & 1 \\
\hline Coryphorus aquilus Peters, 1981 & & 2 & & & & 4 & 1 & 1 & & & 1 & 5 & 14 \\
\hline Cryptonympha copiosa Lugo-Ortiz \& McCafferty, 1998 & & 1 & & & 2 & & 2 & 4 & 7 & 18 & 9 & 2 & 45 \\
\hline Farrodes sp. & 11 & 2 & & & & & 1 & & 2 & & & 12 & 28 \\
\hline Fittkaulus sp. & 9 & 6 & & & 1 & & 40 & 16 & 13 & 22 & & & 107 \\
\hline Hagenulopsis sp. & 1 & & & & & & & & & & & 1 & 2 \\
\hline Harpagobaetis gulosus Mol, 1986 & & & & & & & & & & & & 1 & 1 \\
\hline Hydrosmilodon gilliesae Thomas \& Peru, 2004 & 9 & 3 & & & & 2 & & & & 1 & & & 15 \\
\hline Michophebia surinamenses Savage \& Peters, 1983 & & & 2 & & & & 2 & 9 & 32 & 18 & 1 & & 64 \\
\hline Microphlebia sp. & 3 & & & & & 1 & & 8 & & & & & 12 \\
\hline Miroculis sp. 1 & 1 & & 3 & & & 3 & 2 & 35 & & 1 & & 1 & 46 \\
\hline Miroculis sp. 2 & 5 & 9 & 1 & & & 19 & 2 & 7 & 1 & & & 4 & 48 \\
\hline Paracloeodes binodulus Lugo-Ortiz \& McCafferty, 1996 & & & & & & & 2 & & & & & & 2 \\
\hline Paramaka convexa (Spieth, 1943) & & 1 & & & & & & & & & & & 1 \\
\hline Simothraulopsis sp. & 10 & 3 & 10 & & & & 7 & 17 & 43 & 9 & & 6 & 105 \\
\hline Thraulodes cochunaensis Dominguez, 1987 & 2 & 4 & & & & & & & & & & & 6 \\
\hline Traverhyphes sp. 1 & 1 & 2 & & & & 2 & & & & & & 1 & 6 \\
\hline Traverhyphes sp. 2 & 3 & & & & & & & & & & & & 3 \\
\hline Tricorythodes hiemalis Molineri, 2001 & & & & & & 3 & 1 & 20 & & 15 & & 1 & 40 \\
\hline Tricorythodes sp. 1 & & & & & 1 & 1 & 2 & 2 & & & & & 6 \\
\hline Tricorythodes sp. 2 & & & & & & & 1 & & & & & & 1 \\
\hline Tricorythopsis sp. & & & & & & & 1 & & & 1 & & & 2 \\
\hline Ulmeritoides flavopedes (Spieth, 1943) & 1 & 1 & 28 & 1 & 1 & 1 & 17 & 21 & 6 & 3 & & & 80 \\
\hline Waltzoyphius roberti Thomas \& Peru, 2002 & 1 & 2 & 1 & & & & 3 & & 1 & 7 & 1 & 5 & 21 \\
\hline Zelusia principalis Lugo-Ortiz \& McCafferty, 1998 & 26 & 1 & & & & 1 & 1 & & & & & & 29 \\
\hline Total de indivíduos & 128 & 67 & 104 & 11 & 57 & 54 & 125 & 259 & 226 & 227 & 36 & 62 & 1356 \\
\hline Total de táxons & 23 & 19 & 13 & 6 & 10 & 17 & 25 & 19 & 17 & 16 & 6 & 16 & \\
\hline
\end{tabular}


Na terceira divisão, o RISUZ se separou dos outros ambientes lóticos, que tiveram como indicador Traverhyphes sp.1, enquanto que o RID se separou dos demais semi-lênticos e teve Caenis pflugfelderi Malzacher, 1990 como espécie indicadora.

A DCA aplicada aos dados de presença e ausência explicou, com os dois primeiro eixos, $56 \%$ (eixo $1=0,377$; eixo $2=0,183$ ) da variabilidade total da composição. Nesta análise foram interpretados os escores somente para os dois primeiros eixos, os quais contêm a maior parte da informação (Fig. 4).

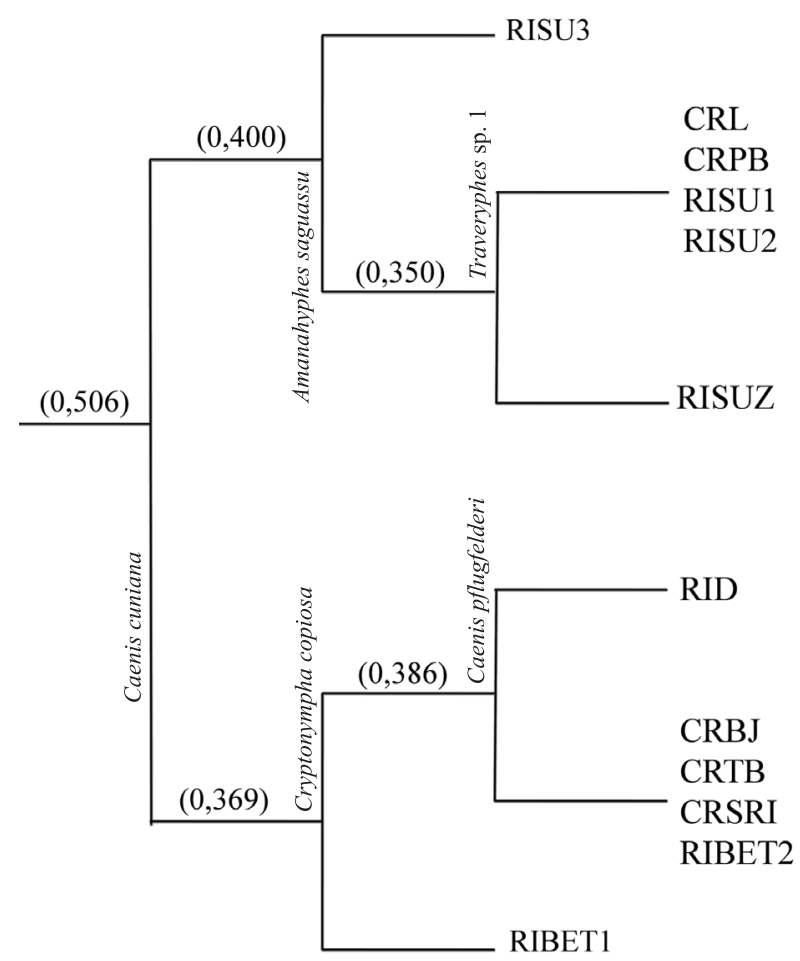

Figura 3. Dendograma ilustrando a classificação dos córregos/rios da bacia do rio Suiá-Miçú pelo método TWINSPAN, de acordo com a composição e abundância das espécies amostradas em 2007 e 2008. As espécies são indicadoras do referente grupo.

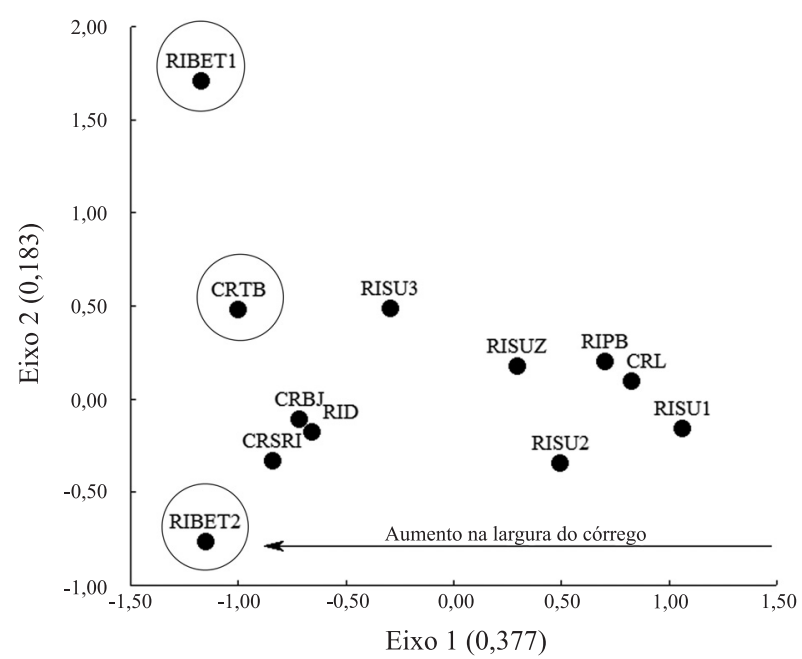

Figura 4. Ordenação da análise de correspondência destendenciada (DCA) a partir da composição e presença/ausência das espécies de Ephemeroptera pelos corpos d'água da bacia do rio Suiá-Miçú, MT, coletados em 2007 e 2008. Os círculos representam os ambientes alterados.
Os ambientes lóticos e de pequeno porte (RISU2, RIPB, CRL e RISU1) tiveram os maiores escores enquanto que os ambientes alterados (RIBET1, RIBET2 e o CRTB) apresentaram os escores mais baixos no primeiro eixo. Os ambientes semi-lênticos alagáveis (RID, CRBJ, CRSRI) e os ambientes lóticos de grande porte, RISUZ e RISU3 (> $16 \mathrm{~m}$ ) apresentaram valores intermediários.

É possível perceber uma variação na composição de espécies conforme ocorre um aumento do canal dos córregos, no qual os ambientes lóticos de pequeno porte possuem uma composição mais similar aos lóticos de grande porte e este, por sua vez, mais similar aos semilênticos alagáveis. Os ambientes alterados lóticos (RIBET1) e semi-lênticos (RIBET2 e CRTB) (destacados na Fig. 4) apresentaram composição de espécies mais similar aos ambientes semi-lênticos alagáveis.

\section{DISCUSSÃO}

A distribuição e composição das comunidades de Ephemeroptera da bacia do Suiá-Miçú apresentaram grande similaridade com os padrões encontrados em outros estudos. Bispo \& Oliveira (1998), trabalhando em córregos de Goiás, também encontraram Leptophlebiidae e Baetidae como as famílias mais abundantes de Ephemeroptera, assim como nos trabalhos de BISPO et al. (2006) e BisPo \& OLIVEIRA (2007), onde estas famílias foram mais ricas.

A maior abundância e riqueza das duas famílias pode ser reflexo da maior diversidade das mesmas, que comportam mais de 26\% (Leptophlebiidae) e $30 \%$ (Baetidae) da diversidade específica total dos Ephemeroptera do Brasil (SAlles, 2010). A baixa abundância e riqueza de Coryphoridae e Euthyplociidae também podem ser explicadas pela suas baixas diversidades, cada uma com menos de $1 \%$ da diversidade total de espécies.

Por serem ambientes alagados, RID, CRSRI e CRBJ, o tipo de vegetação predominante é de Mauritia flexuosa (buriti), e não possuem sombreamento de mata ciliar propriamente dita. Dessa forma, nesses ambientes há maior entrada de luz no meio aquático, proporcionando uma elevada produção autóctone, com a presença de grandes quantidades de macrófitas aquáticas e subaquáticas e depósito de material alóctone carreado que, em consequência da baixa correnteza, acaba depositado nesses locais, resultando em uma maior oferta de alimento (FrANCISCHETTI et al., 2004). Desse modo, a comunidade heterotrófica dessas áreas dispõem não somente de material alóctone, mas também matéria orgânica e algas que afloram com a abertura do dossel (KIKUCHI \& UIEDA, 1998), fato que deve ter contribuído para a maior abundância nestes ambientes.

A maior riqueza de espécies e/ou morfoespécies registrada no RISU1, RISUZ e RISU2 provavelmente está relacionada ao fato desses locais serem ambientes de médio a grande porte com entrada de luz e produção autóctone, o que aumenta a disponibilidade de alimento (VANNOTE et al., 1980), e por serem ambientes tipicamente lóticos. Este resultado corrobora o trabalho de FiDELIS et al. (2008), que registraram a maior riqueza de insetos aquáticos em áreas de correnteza. 
A correnteza é essencial por transportar a matéria orgânica particulada e pequenos insetos que são consumidos pelas larvas (STehr, 1987). Para os efemerópteros, o carreamento de matéria orgânica em suspensão de trechos a montante serve de alimento para as espécies com pouca capacidade de locomoção (LAMPERT \& Sommer, 2007). Segundo Silveira (2004), os processos ecossistêmicos de transporte de energia e ciclagem de matéria são bastante influenciados pelas condições de fluxo do rio.

A menor riqueza registrada no RIBET1 e CTBJ está relacionada às alterações na mata ciliar, que refletiram diretamente nas comunidades, uma vez que a homogeneização dos hábitats diminui a área de ocorrência desses indivíduos (Voelz \& McArthur, 2000). Em ambientes heterogêneos, os hábitats são variados e há uma chance maior das espécies encontrarem um nicho ecológico. Por outro lado, em ambientes alterados pela retirada da vegetação ripária, as chuvas provocam o assoreamento do leito, aumento da quantidade de sedimentos em suspensão e da turbidez, diminuição da velocidade da correnteza e homogeneização de hábitat (Callisto et al., 2001; Goulart \& Callisto, 2003; Moulton \& Souza, 2006). De acordo com Bispo \& Oliveira (2007), em córregos alterados existe uma diminuição dos táxons sensíveis e um aumento significativo em táxons tolerantes, o que ocasiona a redução da diversidade.

Em ecossistemas aquáticos, uma das características mais importantes para determinar a composição de espécies está no tipo de ambiente, não somente pela intensidade do fluxo, mas também pelas características físicas e biológicas associadas (RIBERA, 2008). Muitos macroinvertebrados aquáticos são habitantes de ambientes lênticos ou lóticos, com poucas espécies aptas a viver nos dois tipos de ambientes (RIBERA \& VogLER, 2000). Assim, há grande risco à biodiversidade de macroinvertebrados, dado a frequência cada vez maior do represamento dos cursos d'água para a construção de barragens ou de grandes reservatórios para o fornecimento de energia.

$\mathrm{O}$ fato do RIBET1 ter proximidade com a represa pode influenciar a composição de espécies, pois aquelas adaptadas a ambientes lênticos possuem maior capacidade de dispersão do que as adaptadas a ambientes lóticos (Ribera \& Vogler, 2000; Ribera, 2008), o que pode ter ocasionado a similaridade entre a composição deste local com os demais locais semi-lênticos. De fato, todas as espécies de Ephemeroptera amostradas neste ponto, com exceção de Simothraulopsis sp., cujo conhecimento a respeito de sua biologia é praticamente nulo, são características de ambientes lênticos ou ocorrem em remansos de ambientes lóticos (SAVAGe \& Peters, 1978; DomínguEZ et al., 2006).

As alterações ambientais (retirada da vegetação ripária, represamento e pisoteio de gado) ocorrentes nos RITET2 e CRTB foram as responsáveis na diferenciação da composição de espécies nestes locais. Ao estudar a influência da degradação ambiental, Buss et al. (2002) concluiu que a perda da integridade é o fator mais importante na redução de riqueza de táxons de macroinvertebrados aquáticos.
O RID é o ambiente mais profundo dentre os locais amostrados, com cerca de sete metros, e é o maior em extensão de alagamento dentre os ambientes semilênticos estudados, o que deve refletir na maior heterogeneidade de hábitats neste ambiente, ocasionando a separação dos outros córregos semilênticos.

O RISU3 se diferencia dos demais ambientes lóticos provavelmente devido à largura de seu leito, com aproximadamente $60 \mathrm{~m}$, enquanto que os outros ambientes possuem larguras menores que $15 \mathrm{~m}$. Essa mesma característica pode explicar a separação observada no RISUZ na terceira divisão, pois este rio é cerca de seis metros mais largo do que os outros ambientes (que variam entre 2,53 e 8,17 m).

Estes dados, somados ao resultado do DCA sugerem que a composição de espécies de ambientes lóticos varia em função da largura do canal dos córregos. A teoria de continuidade de rios, proposta por VANNOTE et al. (1980), afirma que os organismos aquáticos se ajustariam à disponibilidade de alimento ao longo do contínuo de um rio de acordo com sua categoria funcional. O tipo de alimento disponível, assim como outras características (temperatura e tipo de substrato) dependeriam da largura do canal do rio, que permite ou não a entrada de matéria orgânica no sistema (em trechos de cabeceira) e de luz (em trechos intermediários e baixos dos rios). Este resultado corrobora o trabalho de Heino et al. (2005), no qual o tamanho do córrego foi o principal fator influenciando a estrutura taxonômica de comunidades de macroinvertebrados. Além disso, com o aumento da largura, grandes rios funcionariam como sistemas lênticos, uma vez que grandes volumes de água acabariam homogeneizando o sistema e reduzindo o número de microhábitats (JUEN et al., 2007).

Os planos de conservação de córregos podem ser baseados em classes de tamanho, pois a largura do córrego é claramente uma característica chave do gradiente para determinar a diversidade taxonômica de comunidades de macroinvertebrados (HeINo et al., 2005).

Os resultados deste estudo mostraram um padrão na distribuição dos efemerópteros imaturos, os ambientes semi-lênticos foram os mais abundantes, já os ambientes médios e grandes, lóticos, foram os mais ricos. As desestruturações físicas dos ambientes aquáticos afetaram a composição de espécies de Ephemeroptera e ocasionaram a perda de espécies e queda na abundância de indivíduos. A largura dos ambientes, alterações no fluxo de água (represas) e características do fluxo da água (lótico/lêntico) foram bons preditores para a composição de espécies de Ephemeroptera da Bacia do Suiá-Miçú, Mato Grosso. Dessa forma, sugere-se a interferência urgente para reconstituição das APPs nas cabeceiras da bacia do rio Suiá-Miçú para a manutenção e reestruturação da diversidade local.

Agradecimentos. Ao Herson Souza Lima e equipe do Laboratório de Entomologia de Nova Xavantina/UNEMAT, pela ajuda em campo. Ao CNPq pelo fomento (proc.n ${ }^{\circ}$ 520268/20059). Aos parceiros EMBRAPA, ISA e ONGs relacionadas à Campanha Y Ikatu Xingu, motivadora deste projeto. Aos revisores anônimos, pelas críticas e sugestões à primeira versão do trabalho. Leandro Juen é bolsista da Capes e agradece a essa agência pelo apoio. 


\section{REFERÊNCIAS BIBLIOGRÁFICAS}

Barber-James, H. M.; Gattolliat, J. L.; Sartori, M. S. \& Hubbard, M. D. 2008. Global diversity of mayflies (Ephemeroptera, Insecta) in freshwater. Hydrobiologia 595:339-350.

Bispo, P. C. \& Oliveira, L. G. 1998. Distribuição espacial de insetos aquáticos (Ephemeroptera, Plecoptera e Trichoptera) em córregos de cerrado do Parque Ecológico de Goiânia, Estado de Goiás. In: Nessimian, J. L. \& Carvalho, A. L. eds. Ecologia de Insetos Aquáticos. Rio de Janeiro, PPGE-UFRJ. Oecologia Brasiliensis 5:191-207.

2007. Diversity and structure of Ephemeroptera, Plecoptera and Trichoptera (Insecta) assemblages from riffles in mountain streams of Central Brazil. Revista Brasileira de Zoologia 24(2):283-293

Bispo, P. C.; Oliveira, L. G.; Bini, L. M. \& Sousa, K. G. 2006. Ephemeroptera, Plecoptera and Trichoptera assemblages from riffles in mountain streams of Central Brazil: Environmental factors influencing the distribution and abundance of immature. Brazilian Journal of Biology 66(2B):611-622.

Burnham, K. P. \& Overton, W. S. 1978. Estimation of the size of a closed population when capture probabilities vary among animals. Biometrika 65(3):625-633.

Buss, D. F.; Baptista, D. F.; Silveira, M. P.; Nessimian, J. L. \& Dorvillé, L. F. M. 2002. Influence of water chemistry and environmental degradation on macroinvertebrate assemblages in a river basin in south-east Brazil. Hydrobiologia 481:125-136.

Cabette, H. S. R.; Giehl, N. F. S.; Dias-Silva, K; Juen, L \& Batista, J. B. 2010. Distribuição de Nepomorpha e Gerromorpha (Insecta: Heteroptera) da Bacia Hidrográfica do Rio SuiáMiçu, MT: riqueza relacionada à qualidade da água e do hábitat. In: Santos, J. E.; Galbiati, C. \& Moschini, L. E. orgs. Gestão e educação ambiental: água, biodiversidade e cultura. São Carlos, RiMa. v.2, p.113-137.

Callisto, M.; Moretti, M. \& Goulart, M. 2001. Macroinvertebrados bentônicos como ferramenta para avaliar a saúde de riachos. Revista Brasileira de Recursos Hídricos 6(1):71-82

Colwell, R. K. 2005. Estatistical estimation of species richness and shared species from samples (EstimateS), versão 7.5. Disponível em: <http://viceroy.eeb.uconn.edu/ estimates>. Acesso em: 29.10.2009.

Colwell, R. K.; MaO, C. X. \& Chang, J. 2004. Interpolating, extrapolating, and comparing incidence-based species accumulation curves. Ecology 85(10):2717-2727.

Courtemanch, D. L. 1996. Commentary on the sub-sampling procedures used for rapid bioassessments. Journal of the North American Benthological Society 15(3):381-385.

Crisci-Bispo, V. L.; Bispo, P. C. \& Froehlich, C. G. 2007. Ephemeroptera, Plecoptera and Trichoptera assemblages in two Atlantic rainforest streams, Southeastern Brazil. Revista Brasileira de Zoologia 24(2):312-318.

Dias, L. G.; Molineri, C. \& Ferreira, P. S. F. 2007. Ephemerelloidea (Insecta: Ephemeroptera) do Brasil. Papéis Avulsos de Zoologia 47(19):213-244.

Dodds, W. K. 2002. Freshwater Ecology: concepts and environmental applications. Aquatic Ecology Series. San Diego, Academic. 568p.

Domínguez, E.; Molineri, C.; Pescador, M. L.; Hubbard, M. \& Nieto, C. 2006. Ephemeroptera of South America. Moscow, Pensoft. 646p.

Downes, B. J. \& Reich, P. 2008. What is the spatial structure of stream insect populations? Dispersal behaviour at different life-history stages. In: LANCASTER, R. A. B. ed. Aquatic insects: challenges to populations. London, Proceedings of the Royal Entomological Society of London. p.184-203.

Ferreira-Peruquetti, P. \& De Marco Jr., P. 2002. Efeito da alteração ambiental sobre comunidades de Odonata em riachos de Mata Atlântica de Minas Gerais, Brasil. Revista Brasileira de Zoologia 19(2):317-327

Fidelis, L.; Nessimian, J. L. \& Hamada, N. 2008. Distribuição espacial de insetos aquáticos em igarapés de pequena ordem na Amazônia Central. Acta Amazonica 38(1):127-134
Francischetti, C. N.; Da-Silva, E. R.; Salles, F. F. \& Nessimian, J. L. 2004. A efemeropterofauna (Insecta: Ephemeroptera) do trecho ritral inferior do Rio Campo Belo, Itatiaia, RJ: composição e mesodistribuição. Lundiana 5(1):33-39.

Gotelli, N. J. \& Colwell, R. K. 2001. Quantifying biodiversity: procedures and pitfalls in the measurement and comparison of species richness. Ecology Letters 4(4):379-391.

Goulart, M. D. C. \& Callisto, M. 2003. Bioindicadores de qualidade de água como ferramenta em estudos de impacto ambiental. Revista da FAPAM 2(1):153-164.

Heino, J.; Parviainen, J.; Paavola, R.; Jehle, M.; Louhi, P. \& MuotKA, T. 2005. Characterizing macroinvertebrate assemblage structure in relation to stream size and tributary position. Hydrobiologia 539:121-130.

Hill, M. O. 1979. TWINSPAN- a FORTRAN program for arranging multivariate data in an ordered two-way table by classification of the individuals and attributes. Nova York, Cornnel University. 90p.

Hill, M. O. \& GaUCH, H. G. 1980. Detrended correspondence analysis, an improved ordination technique. Vegetatio 42:47-58.

Juen, L.; Cabette, H. S. R. \& De Marco, P. 2007. Odonate assemblage structure in relation to basin and aquatic habitat structure in Pantanal wetlands. Hydrobiologia 579:125-134.

KIKUCHI, R. M. \& UIEDA, V. S. 1998. Composição da comunidade de invertebrados de um ambiente lótico tropical e sua variação espacial e temporal. In: Nessimian, J. L. \& Carvalho, A. L. eds. Ecologia de Insetos Aquáticos. Rio de Janeiro, PPGEUFRJ. Series Oecologia Brasiliensis. v.5, p.157-173.

LAmpert, W. \& Sommer, U. 2007. Limnoecology. 2. ed., Nova York, Oxford University. 324p.

Melo, A. S. 2008. O que ganhamos "confundindo" riqueza de espécies e equabilidade em um índice de diversidade? Biota Neotropica 8(2):21-27.

Melo, A. S. \& Hepp, L. U. 2008. Ferramentas estatísticas para análises de dados provenientes de biomonitoramento. Oecologia Brasiliensis 12(3):463-486.

Mittermeier, R. A.; Fonseca, G. A. B.; Rylands, A. B. \& Brandon, K. 2005. Uma breve história da conservação da biodiversidade no Brasil. Megadiversidade 1(1):14-21.

Moulton, T. P. \& Souza, M. L. 2006. Conservação com base em bacias hidrográficas. In: Rocha C. F. D.; Bergallo H. G.; Sluys M. V. \& Alves A. S. A. eds. Biologia da conservação: essências. São Carlos, RiMa. p.157-182.

NeU, V. 2006. Inpactos antrópicos nas cabeceiras do Rio Xingu. Disponível em: <http://www.yikatuxingu.org.br/arquivos/projetos/ 35/Estudoporcentagem20Vaniaporcentagem20Neu_CENA/ USP.doc>. Acesso em: 13.10.2010.

Ratter, J. A.; Askew, G. P.; Montgomery, R. F. \& Gifford, D. R. 1978. Observations on the vegetation of northeastern Mato Grosso II. Forest and soils of the Rio Suiá-Miçú area. Proceedings of the Royal Society 293:191-208.

RiberA, I. 2008. Habitat constraints and the generation of diversity in freshwater macroinvertebrates. In: LANCASTER, R. A. B. ed. Aquatic insects: challenges to populations. London, Proceedings of the Royal Entomological Society of London. p. 289-312

Ribera, I. \& Vogler, A. P. 2000. Habitat type as a determinant of species range sizes: the example of lotic-lentic differences in aquatic Coleoptera. Biological Journal of the Linnean Society 71:33-52.

Rosenberg, D. M. \& Resh, V. H. 1993. Introduction to freshwater biomonitoring and benthic macroinvertebrates. In: RoSENBERG, D. M. \& Resh, V. H. eds. Freshwater biomonitoring and benthic macroinvertebrates. New York, Chapman \& Hall. p. $1-9$.

Salles, F. F. 2010. Lista de espécies de Ephemeroptera (Insecta) registradas para o Brasil. Disponível em: <https:/ /sites.google.com/site/listaephemeropteradobrasil /home>. Acesso em: 25.10.2010.

Santos, A. J. 2003. Estimativas de riqueza em espécies. In: CulleN Jr, L.; Rudran, R. \& Valladares-Padua, C. eds. Métodos de Estudos em Biologia da Conservação \& Manejo da Vida 
Silvestre. Curitiba, UFPR, Fundação O Boticário de Proteção à Natureza. p.19-41.

Savage, H. M. \& Peters, W. L. 1978. Fittkaulus maculates, a new genus and species from northern Brazil (Leptophlebiidae: Ephemeroptera). Acta Amazonica 8(2):293-298.

Schwartzman, S. \& Zimmerman, B. 2005. Conservation alliances with indigenous peoples of the Amazon. Conservation Biology 19(3):721-727.

Siegloch, A. E.; Froenlich, C. G. \& Kotzian, C. B. 2008 Composition and diversity of Ephemeroptera (Insecta) nymph communities in the middle section of the Jacuí River and some tributaries, southern Brazil. Iheringia, Série Zoologia, 98(4):425-432.

Silveira, M. P. 2004. Aplicação do biomonitoramento para avaliação da qualidade da água em rios. 1. ed., Jaguariúna, Embrapa Meio Ambiente. 68p.

Stehr, F. W. 1987. Immature Insects. Iowa, Kendall/Hunt Publishing Company. v.1, 754p.

Strahler, H. N. 1957. Quantitative analysis of watershed geomorphology. American Geophysical Union Transactions 38:913-920.

Vannote, R. L.; Minshall, G. W.; Cummins, K. W.; Sedell, J. R. \& Cushing, C. E. 1980. The River Continuum Concept. Canadian Journal of Fisheries and Aquatic Sciences 37:130-137.

Voelz, N. J. \& McArthur, V. 2000. An exploration of factors influencing lotic insect species richness. Biodiversity and Conservation 9:1543-1570. 